\section{Sanofi's dengue vaccine rounds final corner}

An approval for a first dengue vaccine seems in sight, even if the efficacy data are not as strong as was once hoped.

\section{Asher Mullard}

Sanofi has recently completed two Phase III trials for its dengue virus vaccine, and a regulatory filing is expected shortly. For the 2.5 billion people across more than a hundred countries who are at risk of the mosquito-borne disease - which is the world's fastest-spreading tropical disease an effective treatment can't come soon enough. But uncertainty still remains about how well Sanofi's CYD-TDV, also known as ChimeriVax-Dengue, will fit the bill.

"In a nutshell, the data that Sanofi has released look encouraging," says Joachim Hombach, Senior Adviser at the Department of Immunization, Vaccines and Biologicals at the World Health Organization and a member of the Dengue Vaccine Initiative. The results seem broadly consistent across the clinical trials, he says, and assuage long-standing safety concerns surrounding dengue vaccines. Because patients with naturally-acquired immunity to any one strain of the virus are at higher risk of severe disease if they are infected by a second strain, researchers have worried that vaccines would carry high risks. CYD-TDV - a live attenuated vaccine that combines four chimeric yellow-fever vaccine viruses that express the genes from the four dengue serotypes - has yet to raise any safety flags. "[Safety] has been in the past a major obstacle. I think the data that we have are really from that perspective reassuring."

The vaccine's efficacy profile, however, is less clear-cut. Sanofi's latest data show that in the 20,000-patient Central and South American Phase III trial, the vaccine provided $50 \%, 74 \%$ and $77 \%$ protection against serotypes 1, 3 and 4, respectively, but only $42 \%$ protection against serotype 2 . Sanofi's head of dengue research Nick Jackson sees these results as in line with the 10,000-patient Asian Phase III trial, in which the vaccine provided protection of $55 \%, 65 \%$ and $72 \%$ against serotypes 1,3 and 4 , but only $35 \%$ against serotype 2 (Capeding, M. R. et al. Lancet 11 Jul 2014). Both trials provided better efficacy results than did the proof-of-concept Phase Ilb trial, in which the vaccine provided an overall efficacy of $32 \%$, dragged down by a $9.2 \%$ protection against serotype 2 (Lancet 380, 1559-1567; 2012). Because all four serotypes circulate and lead to clinical disease, the lower efficacy against serotype 2 in particular poses a problem.

Sanofi will present detailed data from the latest trial at the American Society for Tropical Medicine and Hygiene meeting this November, ahead of an anticipated filing next year. The community is looking forward to seeing whether there is heterogeneity in the data - for instance, an increased efficacy in older children, as was seen in the Phase III Asian population - and to assessing longer-term follow-up data on the durability of the response.

But, even assuming an average protection of $56-60 \%$, would the vaccine be useful? "That's a tough question," says Hombach. "A $60 \%$ effective vaccine could be of utility, there is absolutely no doubt. But what needs to be done with a moderately effective vaccine is that you need to consider very carefully the risk and benefits and you need to look at the cost-effectiveness of such a measure. It is a complex estimation that a country would need to go through."

Jackson counters that "in the absence of any specific treatment or effective prevention, the ability to provide efficacy to young kids, I would argue, is a significant public health benefit". He adds that the Phase III data show the vaccine reduces hospitalization by as much as $80 \%$, a major win given that hospitals can become overpacked during an epidemic.

Annelies Wilder-Smith echoed these sentiments in a recent article (Wilder-Smith, A. Lancet 11 Jul 2014).

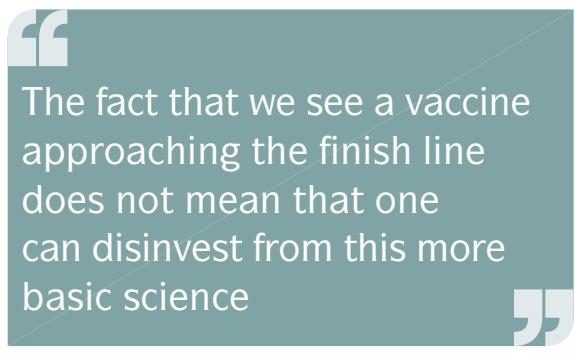

"With an estimated 96 million clinically apparent dengue infections annually, a reduction by half would present a substantial public health benefit that would support vaccine introduction," she wrote. However, it "might be of limited use in countries with low dengue endemicity, or in international travellers from non-dengueendemic countries," she adds, and notes that "with a $56 \%$ efficacy this vaccine will never be a single solution." Wilder-Smith was an investigator in a Phase II trial of the vaccine.

\section{Dissecting the dengue response}

Sanofi is still trying to figure out exactly why their vaccine disappointed on serotype 2 .

And the discrepancy in efficacy between the Phase IIb and Phase III data suggests that variability in viral genetics might be an important part of this. But the company's development of the vaccine has yielded one other important lesson: the neutralizing antibody assays that are typically used as a surrogate measure of vaccine efficacy don't work very well for dengue virus. CYD-TDV scored highly on this assay for all four serotypes. "This is an intriguing finding that makes vaccine development much more complicated," says Hombach. "The question now is "can we come up with an assay that is better that you can also use in large clinical trials?"'

Sanofi and others are working on alternative assays. "Realistically, I think we are 1 or 2 years away from having those batteries of new assays," says Jackson.

Sanofi's development track has also highlighted how little is still known about the immune response to dengue virus. In particular, there is a need for more research into the $T$ cell responses and the humoral responses to primary and secondary infection, says Hombach. "The fact that we see a vaccine approaching the finish line does not mean that one can disinvest from this more basic science," he says.

Several companies could also benefit from more basic research. Five other tetravalent dengue vaccines are in clinical trials, including two in Phase II trials. "It's good that we have a pipeline," notes Hombach. 
Not only might alternative vaccines have more favourable profiles, but they could be good for competition, access and affordability.

Takeda's Phase II candidate TAK-003, also known as DENVax, combines a live attenuated strain of serotype 2 dengue plus chimeric strains of this serotype that are modified to express structural genes from the other serotypes. In July, 96-patient Phase I data showed that this vaccine elicited neutralizing antibodies against all four serotypes, with $96 \%$ of participants seroconverting to three or more dengue viruses (Lancet Infect. Dis. 14, 830-838; 2014). But in light of Sanofi's experience, seroconversion alone is not as encouraging as it used to be.

The US National Institutes of Health and the Brazilian Instituto Butantan's Phase II TetraVax-DV, also known as

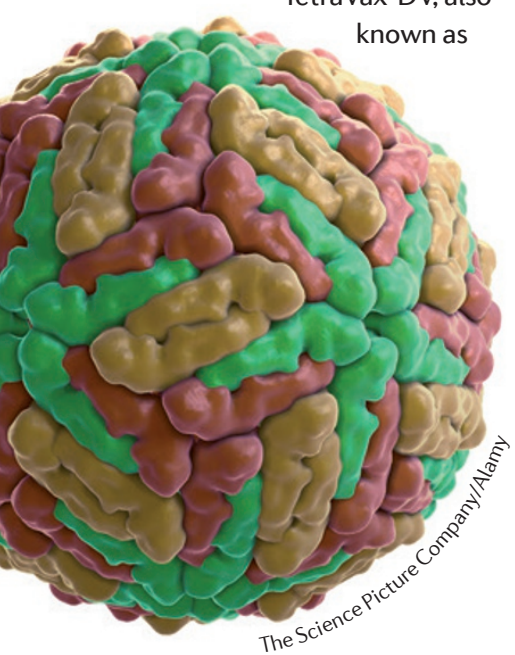

TV-003, combines the four live attenuated dengue serotypes (). Infect. Dis. 207, 957-965; 2013).

"These are very respectable candidates," says Hombach. "You could say these attenuated vaccines might have certain advantages over Sanofi's vaccine in the sense that they are fully dengue. But this is an entirely theoretical argument; we cannot predict any superiority of these vaccines vis-à-vis Sanofi's vaccine at this stage," he adds.

Several other tetravalent approaches are also being trialled in Phase I. Merck \& Co.'s V180 is a subunit vaccine; GlaxoSmithKline and the Walter Reed Army Institute of Research's DPIV-001 is a purified inactivated tetravalent vaccine; and the US Naval Medical Research Center and Vical's Vaxfectin is a DNA vaccine.

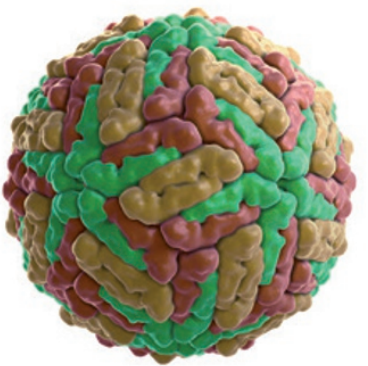

